\title{
FACTEURS ÉCOLOGIQUES ET QUALITÉ DU BOIS
}

\author{
H. POLGE \\ Station de Recherches sur la Qualité des Bois, \\ Centre national de Recherches forestières, I. N. R. A., \\ Champenoux 54370 Einville
}

\section{RÉSUMÉ}

L'effet des facteurs écologiques sur la qualité du bois est tel que les utilisateurs de produits forestiers eux-mêmes y sont sensibles et que l'on a pu parler de "crus" de bois :

- cas du chêne rouvre,

- cas du chêne pédonculé.

Parfois même, l'effet «station » prime l'effet « espèce » :

- cas des bois du Nord,

- cas des bois d'altitude.

Mais, en règle générale, l'effet « espèce » demeure important pour la qualité du bois, même s'il est peu marqué sur la croissance (comparaison douglas — grandis).

A l'opposé, il arrive que les facteurs écologiques n'exercent qu'une influence limitée sur la valeur technologique, non seulement pour une espèce, mais même pour un groupe d'espèces (cas des pins à cinq feuilles).

La station ne « marque » pas que les caractéristiques classiques : densité, texture, longueur de fibres; elle influe aussi sur d'autres critères qualitatifs :

- perméabilité du douglas,

- cœur rouge du hêtre.

Les exemples pris par l'auteur sont les suivants :

\section{1 - Influence des facteurs climatiques}

- Variations de la qualité du bois de Fagus orientalis en fonction de l'altitude,

- Influence du vent sur la qualité du bois de Pin maritime des Landes

- Expérience d'irrigation sur Pins sylvestres,

- Xyloclimatologie.

\section{2 - Influence des facteurs édaphiques}

- Expérience sur pins maritimes en Tunisie,

- Effets séparés du sol et de l'hérédité sur les caractéristiques juvéniles du bois de hêtre,

- Comparaison de qualité du bois de sapin pectiné sur roche-mère gréseuse et sur roche-mère granitique. 


\section{1. - INTRODUCTION}

Que les facteurs écologiques puissent exercer une influence sur la qualité du bois, cela peut sembler une évidence pour qui sait à quel point les utilisateurs de produits forestiers peuvent avoir des préférences pour telle forêt, tel canton de forêt, ou même telle parcelle, préférences qui se traduisent par des prix de vente beaucoup plus élevés.

Dans le cas de forêts ou de parties de forêts très étendues, on peut penser à une influence possible d'autres facteurs, comme le traitement ou l'hérédité; en revanche, lorsqu'un peuplement réputé ne représente qu'une petite surface noyée au sein d'un massif important dont rien ne le distingue à première vue, on est bien obligé de penser à une influence prépondérante de la station, et notamment des facteurs édaphiques; on en arrive alors à parler dans certains cas de « crus » de bois, par analogie avec tel ou tel vignoble réputé qui ne se différencie de ses voisins que par la très haute qualité de sa production.

Le cas du Chêne rouvre est particulièrement typique à cet égard : si la sylviculture conditionne dans une large mesure la qualité de son bois, l'effet des facteurs écologiques ne doit pas être sous-estimé pour autant puisque, toutes choses égales par ailleurs, les chênes des stations pauvres à accroissements fins sont en général des chênes tendres, à faible texture et à aubier mince; mais, parmi tous les peuplements de Chêne de qualité, certains jouissent d'un prestige inégalé, grâce à telle ou telle caractéristique, comme la couleur du bois de cœur, dont on peut penser qu'elle dépend, elle aussi, d'un facteur écologique particulier, par exemple la présence dans le sol d'un oligo-élément favorable.

Pour le Chêne pédonculé également, il existe, à côté de nombreux peuplements à croissance relativement rapide qui ne fournissent qu'un bois assez médiocre, parce que dur et nerveux, des stations privilégiées où l'on trouve des bois à accroissements tout aussi larges, mais de bien meilleure qualité grâce à la faible densité de son bois d'été; tel est le cas en particulier des Chênes de Bourgogne qui, poussant sur les alluvions récentes de la vallée de la Saône, c'est-à-dire sur un sol frais, profond et assez riche, étant en outre soumis, du fait d'un climat relativement chaud, à une forte transpiration estivale, ont une zone de bois final bien pourvue en petits vaisseaux et en parenchyme périvasculaire, mais pauvre en fibres, donc relativement tendre.

L'influence des facteurs écologiques sur la qualité du bois est parfois telle que l'effet « station» en arrive à primer l'effet « essence »; cela se produit notamment pour les bois résineux en provenance des forêts nordiques, épicéas et pins sylvestres, qui se ressemblent beaucoup plus entre eux qu'ils ne ressemblent les uns et les autres aux bois de même espèce poussant en climat tempéré (fig. 1); les bois du Nord ne se caractérisent pas seulement par des accroissements très fins et très réguliers; ils se différencient également des autres par un bois final extrêmement mince et de plus formé de fibres à cavités cellulaires relativement larges, ce qui tient sans doute à la très courte durée de la saison de végétation et à un régime photopériodique très particulier caractérisé par la longueur du jour pendant la période de croissance. On peut observer incidemment que les caractéristiques des bois du Nord ne sont pas toutes favorables, puisque la faible densité du bois d'été entraîne une baisse de résistance mécanique; ainsi, certaines publicités, comme celle qui est faite en faveur de l'utilisation des bois du Nord pour les charpentes lamellées collées, reposent sur une méconnaissance des relations entre facteurs écologiques et qualité du bois. 

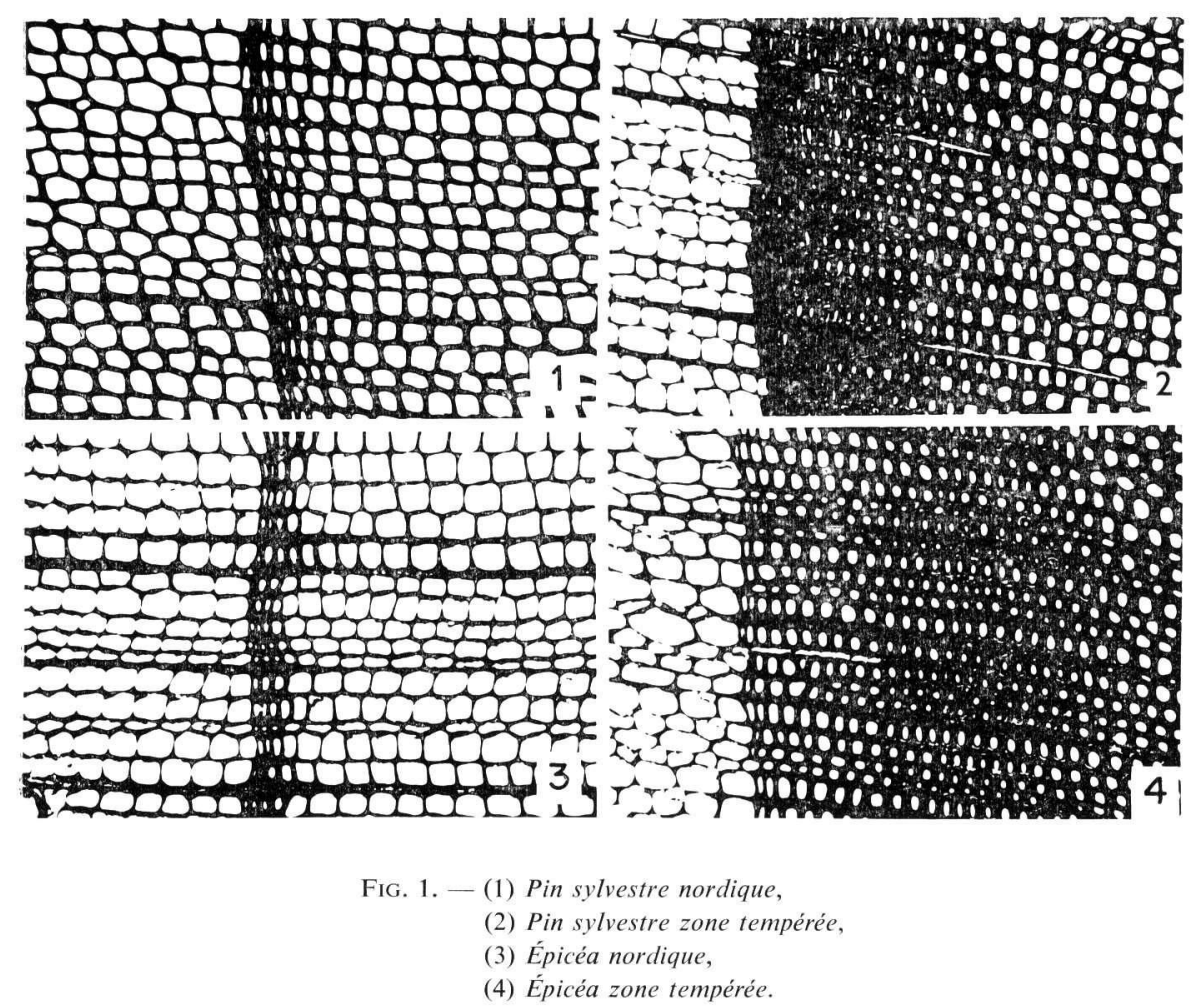

Ce qui est vrai pour les bois du Nord l'est également pour les bois poussant en région tempérée, mais à une altitude élevée : épicéas, mélèzes et pins noirs des forêts de haute montagne ont entre eux beaucoup de similitudes, mais en revanche diffèrent considérablement des bois de mêmes espèces cultivés en plaine.

En dehors de ces cas extrêmes, l'effet « espèce » est important et se traduit en fait par une aptitude héréditaire à produire telle ou telle catégorie d'éléments anatomiques suivant un schéma général intangible, mais avec des modalités (proportion relative, rythme de production, caractéristiques dimensionnelles) qui sont fonction, entre autres choses, de l'environnement.

La comparaison d'échantillons de Douglas et d'Abies grandis (fig. 2) poussant dans des conditions identiques au point de vue station et concurrence est, à cet égard, instructive : les deux espèces réagissent aux variations de climat d'une année à l'autre de façon semblable (effet « facteurs écologiques ») mais différente (effet « espèce ») : les accroissements annuels varient pareillement d'une saison de végétation à l'autre, et on peut observer de grandes similitudes dans la largeur du bois d'été (1949 par exemple) et même dans la production des faux cernes (1947); mais, pour chaque année, la proportion de bois final et la densité propre de celui-ci sont plus élevées chez le Douglas que chez le Grandis, d'où découlent tout naturellement des différences importantes au point de vue qualité du bois. 


\section{* 14.9 GRANDIS}

\section{PEZANIN}

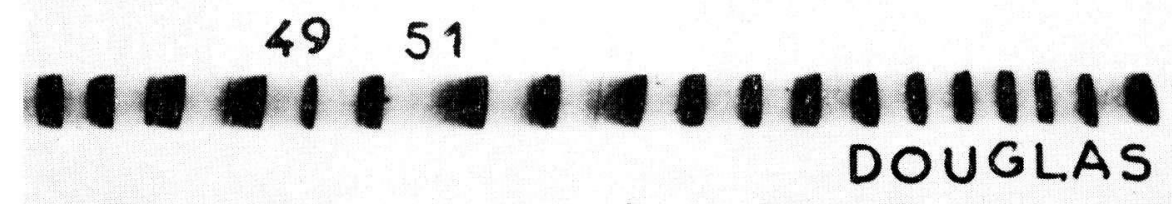

FIG. 2. - Effets des variations climatiques annuelles sur le grandis et le douglas

Il existe cependant des cas où l'influence des facteurs écologiques sur la valeur technologique des produits est très limitée, non seulement pour une espèce déterminée, mais encore pour tout un groupe d'espèces : ainsi, le bois de tous les pins à cinq feuilles est, en règle générale, caractérisé par une faible densité, une faible rétractibilité et une très bonne homogénéité, qu'il s'agisse du Pin cembro à croissance extrêmement ralentie poussant dans l'étage subalpin, du Pin weymouth à accroissement moyen des forêts sur sol convenablement drainé de son aire d'origine, ou du même Pin weymouth à croissance rapide introduit en France dans des stations le plus souvent très humides, du Pin de l'Himalaya (Pinus excelsa), ou encore du Pinus aristata que l'on trouve en bordure du désert de l'Arizona.

Une littérature abondante a été consacrée aux relations entre facteurs écologiques et qualité du bois dont il existe d'excellentes synthèses (voir notamment Dadswell 1960, Hildebrandt 1960, ou encore LARSON 1962).

Je ne retiendrai que deux exemples :

- le premier montre, qu'en dehors des caractéristiques classiques : densité, pourcentage de bois final, longueur de fibres,... il existe d'autres critères de qualité, auxquels on ne pense pas a priori, mais qui sont cependant, eux aussi, sous une dépendance étroite des facteurs de l'environnement; ainsi, Scott et Stamm (in Dadswell, 1960 op. cit.) ont pu établir que la perméabilité du Douglas (qui joue un rôle très important dans l'application des traitements antiseptiques) diminue significativement avec l'altitude, en liaison vraisemblablement avec une adaptation des ponctuations à une transpiration accrue,

— du deuxième, on doit tirer une leçon : pour connaître de façon sûre l'influence des facteurs écologiques sur la qualité du bois, il ne suffit pas de se fier à des impressions plus ou moins subjectives, mais il faut procéder à des expérimentations sérieuses basées sur un grand nombre d'échantillons. C'est ce que prouve l'étude du cœur rouge du Hêtre dont on connaît les incidences technologiques défavorables : après avoir analysé plus de 41000 arbres, KNIGGE (1961) a prouvé que cette maladie était plus fréquente sur muschelkalk que sur grès bigarré ou sur limon, que son importance augmentait avec le degré de décomposition de l'humus, et qu'enfin elle affectait un moins grand nombre de tiges et était de plus petite taille sur les sols humides que sur les sols secs. 


\section{2. - QUELQUES EXEMPLES D'ACTION DES FACTEURS ÉCOLOGIQUES TIRÉS D'EXPÉRIENCES DE LA STATION}

\section{1. - Influence des facteurs climatiques}

\subsection{Variations de la qualité du bois de Fagus orientalis en fonction de l'altitude.}

Dans cette expérience (PARSA, 1970), ont été comparées trois stations situées sur un même versant de l'Elbourz sur une même roche-mère cristalline et sur un même type de sol (sol brun forestier acide à structure argilo-limoneuse caractérisé par une capacité d'échange élevée et une forte teneur en humus).

La sylviculture appliquée aux trois stations étant elle-même identique, la principale source de variation était l'altitude qui variait de 750 à 1000 , puis à 1250 mètres; l'analyse des résultats a fait apparaître des différences significatives globales pour toutes les caractéristiques étudiées : largeur des accroissements, infra-densité, pourcentage de bois de densité égale ou supérieure à $600 \mathrm{~g} / \mathrm{dm}^{3}$, densités maximales et minimales annuelles et hétérogénéité, toutes ces caractéristiques allant en diminuant au fur et à mesure que l'altitude s'élève.

Pour deux des critères en cause : la densité maximale annuelle et l'hétérogénéité, chaque station se différencie même des deux autres au seuil de 5 p. 100; il y a donc un effet « altitude » très net qui fait que la qualité du bois augmente avec l'élévation puisque, pour le Hêtre, une bonne homogénéité et une faible densité sont des facteurs favorables.

On peut penser que, pour Fagus orientalis, et dans les conditions climatiques de l'Elbourz où les précipitations sont relativement élevées, l'évapo-transpiration augmente avec l'altitude, surtout en été, sous l'effet conjugué d'une diminution de la tension de vapeur d'eau dans l'atmosphère, et d'une violence accrue des vents, l'ensemble se traduisant sur le plan ligneux par la production d'un bois final riche en petits vaisseaux.

\subsection{Influence du vent sur la qualité du bois de Pin maritime des Landes.}

Un facteur climatique précis : le vent, est à l'origine de variations importantes des caractéristiques technologiques qui affectent un million d'hectares de forêts landaises, et qui occasionnent une très forte anisotropie Est-Ouest (Polge et ILLY, 1967).

Cette hétérogénéité se traduit par la production sur le côté Est des tiges d'un bois plus dense à accroissements plus larges, à rétractibilité axiale et à teneur en lignines plus élevées, à rendement en fibres plus faible et à plus forte teneur en résine.

En ce qui concerne la densité du bois, qui est le principal facteur d'anisotropie, la supériorité cu côté Est sur le côté Ouest se retrouve dans au moins 95 p. 100 des individus et affecte tous les niveaux de la tige (fig. 3).

La responsabilité du vent dominant d'Ouest dans le phénomène en cause peut être mis en évidence par l'observation de ce qui se passe sur les lisières Ouest et Est d'un même peuplement : 


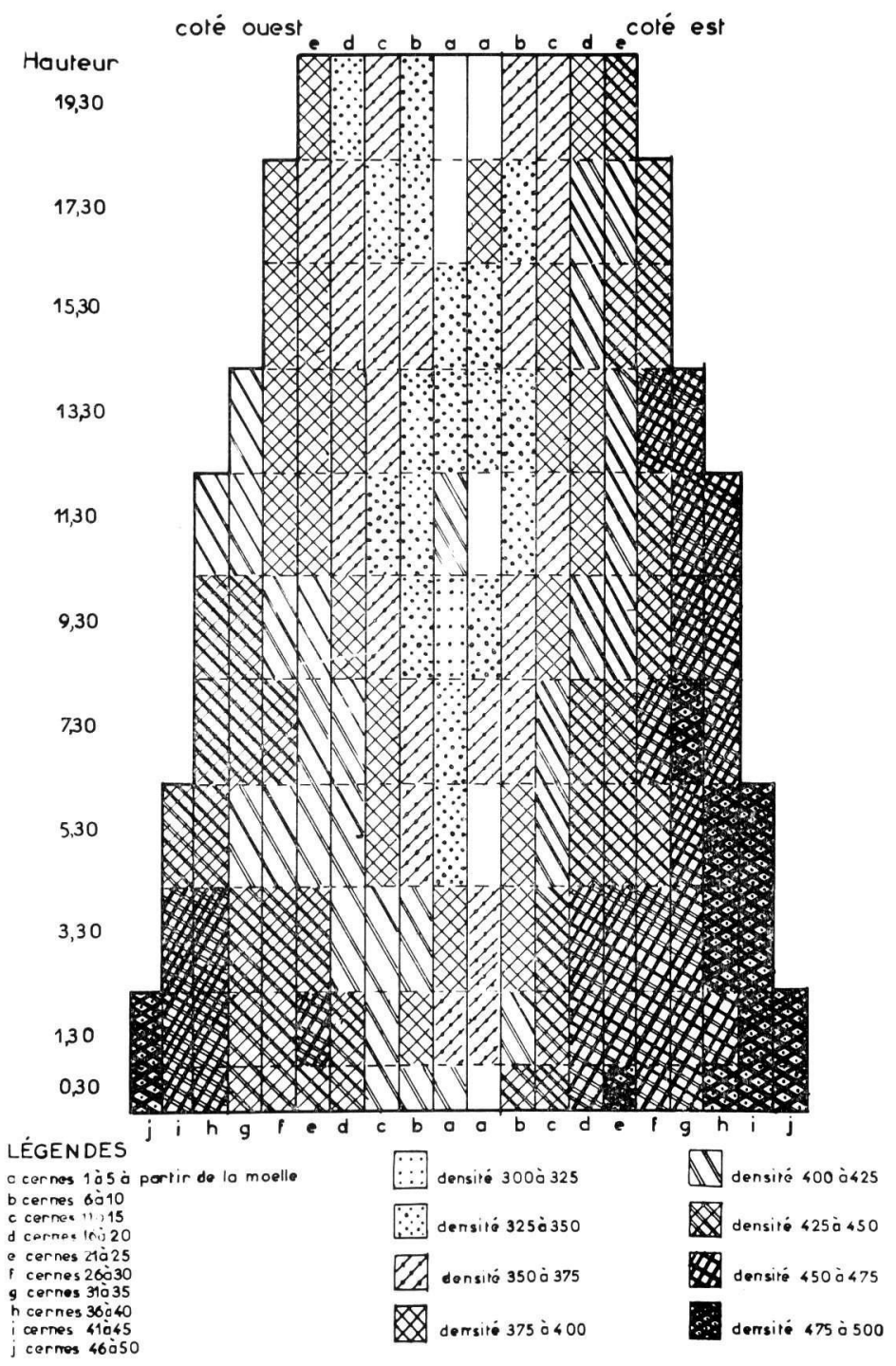

FIG. 3. - Variations de la densité du bois de pin maritime en fonction de la hauteur, de l'orientation et de la distance à la moelle.

Moyennes obtenues sur 20 arbres de la Forêt de l'Hermitage (45 km de l'Océan) 
TABLEAU 1

Lisière EST

\begin{tabular}{|c|c|c|c|c|c|c|c|}
\hline \multicolumn{2}{|c|}{ Densité en $\mathrm{g} / \mathrm{dm}^{3}$} & \multicolumn{2}{|c|}{$\begin{array}{l}\text { Largeur de cernes } \\
\text { en } \mathrm{mm} \times 100\end{array}$} & \multicolumn{2}{|c|}{ Densité en $\mathrm{g} / \mathrm{dm}^{3}$} & \multicolumn{2}{|c|}{$\begin{array}{c}\text { Largeur de cernes } \\
\text { en } \mathrm{mm} \times 100\end{array}$} \\
\hline Est & Ouest & Est & Ouest & Est & Ouest & Est & Ouest \\
\hline 323,3 & 316,2 & 121,3 & 110,3 & 332,2 & 311,5 & 125,7 & 114,9 \\
\hline
\end{tabular}

Lisière OUEST

L'anisotropie des densités (qui est la principale à prendre en considération lorsqu'il s'agit de qualité du bois) est beaucoup plus marquée sur la lisière Ouest exposée aux vents, et la différence est significative au seuil de 5 p. 100.

\subsection{Expérience d'irrigation sur Pins sylvestres.}

Profitant d'un déficit de pluviosité qui s'est maintenu durant tout le début de l'année 1967 et qui s'est aggravé en juin et juillet, un apport d'eau équivalant à $70 \mathrm{~mm}$ de pluie a été réalisé au début du mois d'août, afin de pouvoir étudier les incidences de l'approvisionnement en eau estival sur les caractéristiques du bois (POLge et Keller, 1968).

Les résultats sont consignés dans le tableau 2.

L'année 1966 a été prise comme année de référence, de façon à tenir compte des variations individuelles de vigueur qui auraient pu subsister en dépit du soin pris à équilibrer arbres traités et témoins au sein du dispositif expérimental; on voit que l'apport d'eau effectué a entraîné une augmentation significative de la largeur de l'accroissement (ce qui n'est pas pour surprendre), mais que cette augmentation de largeur n'a concerné que la partie à faible densité de l'accroissement.

On peut en déduire que l'irrigation à laquelle il a été procédé est intervenue durant la phase de croissance correspondant à la formation du bois initial, et tout se passe comme si le traitement appliqué n'avait fait que différer dans le temps les divers phénomènes physiologiques responsables de la formation du bois d'été, sans en altérer l'ampleur ni le rythme.

Ces résultats confirment certaines observations faites sur jeunes semis dans des conditions d'environnement contrôlées : sur Pinus resinosa, Larson (1963) a montré qu'une sécheresse prolongée réduit la vitesse d'élongation des aiguilles, et par suite occasionne une diminution de production d'auxine qui entraîne à son tour la formation de cellules type « bois final »; une irrigation, au contraire, a pour effet de prolonger le fonctionnement des méristèmes terminaux, donc la production d'auxine, et a pour conséquence la formation d'un bois initial plus large; en fait, l'alimentation en eau n'agit qu'indirectement dans le phénomène en cause par l'intermédiaire de l'auxine, car des sujets décapités ne forment que des cellules de bois final, quels que soient les apports d'eau dont ils peuvent bénéficier. 
TABLEAU 2

\begin{tabular}{|c|c|c|c|}
\hline Caractéristiques analysées & $\begin{array}{c}\text { Valeur } \\
\text { moyenne } \\
\text { pour les } \\
16 \text { arbres } \\
\text { traités }\end{array}$ & $\begin{array}{l}\text { Valeur } \\
\text { moyenne } \\
\text { pour les } \\
16 \text { témoins }\end{array}$ & Test «t» \\
\hline 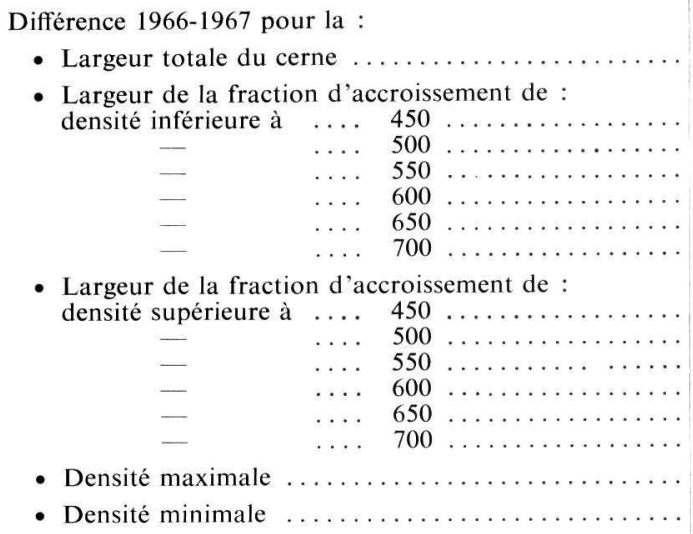 & $\begin{array}{r}-4,67 \\
-1,03 \\
-0,20 \\
-1,45 \\
-2,69 \\
-3,60 \\
-4,84 \\
\\
-3,64 \\
-4,47 \\
-3,22 \\
-1,98 \\
-1,07 \\
+0,17 \\
-21,6 \\
-31,0\end{array}$ & $\begin{array}{r}+5,12 \\
+\quad 5,19 \\
+5,75 \\
+6,56 \\
+7,87 \\
+5,19 \\
+4,94 \\
-0,07 \\
-0,63 \\
-1,43 \\
-2,65 \\
-0,07 \\
+0,19 \\
-24,1 \\
-41,9\end{array}$ & $\begin{array}{l}-2,71^{* *} \\
-2,92^{* *} \\
-2,33^{*} \\
-2,65^{* *} \\
-3,13^{* *} \\
-2,47^{*} \\
-2,68^{* *}\end{array}$ \\
\hline
\end{tabular}

\subsection{Xylochronologie.}

L'étude des variations de densité à l'intérieur des accroissements annuels et entre accroissements, entreprise à l'origine dans un but exclusivement technologique (appréciation quantitative de l'hétérogénéité du bois), a très rapidement fait apparaître des similitudes entre cernes de même année, non seulement au sein d'un même peuplement, mais encore entre stations, même fort éloignées les unes des autres.

Ainsi, les 4 échantillons de Douglas de la figure 4 sont très différents, aussi bien au point de vue origine qu'au point de vue largeur des accroissements, et même densité moyenne; cependant, on peut observer entre eux des analogies frappantes de largeurs de bois d'été, qu'il s'agisse de l'année 1949 à texture anormalement faible ou de l'année 1951 où le pourcentage de bois dense est, au contraire, particulièrement élevé.

Les courbes de variation de la densité permettent tout naturellement de préciser ce phénomène, et de faire apparaître éventuellement soit des similitudes, soit au contraire des dissemblances entre espèces : sur la figure 5, on peut voir que l'année 1953 est caractérisée par un pic unique relativement acéré, aussi bien pour le Douglas que pour le Grandis, qu'en 1954, la montée en densité a été brutale pour les deux espèces, avec une partie dense de l'accroissement se terminant par une sorte de replat; pour toutes deux également, l'année 1960 a une densité maximale inférieure, à la fois à celles de 1959 et 1961; en revanche, Grandis et Douglas se différencient par la forme du profil de densité de 1953, dont la concavité est tournée vers le haut pour le Grandis et au contraire vers le bas pour le Douglas.

Dans plusieurs expériences successives, les analyses statistiques ont montré que les années se différenciaient plus les unes des autres par certains critères qualitatifs tirés des courbes de variation de la densité du bois (densités maximales et minimales annuelles) que 


\section{GRANDIS}

\section{DOUGLAS}

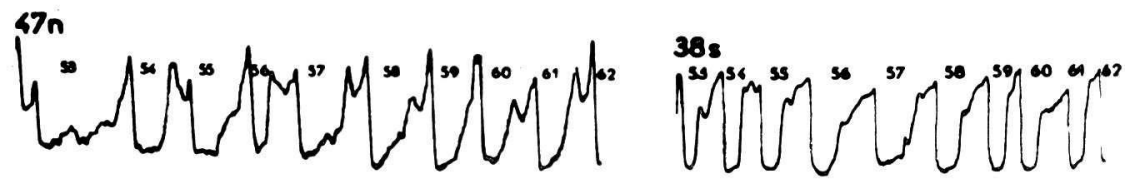
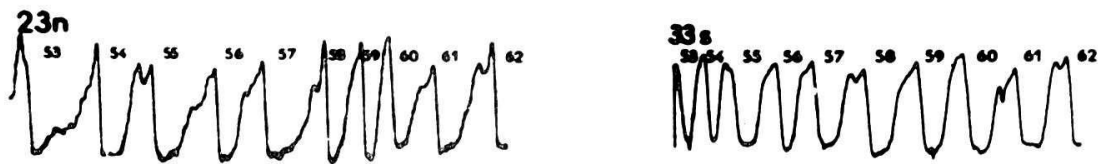

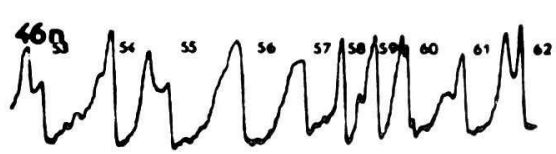
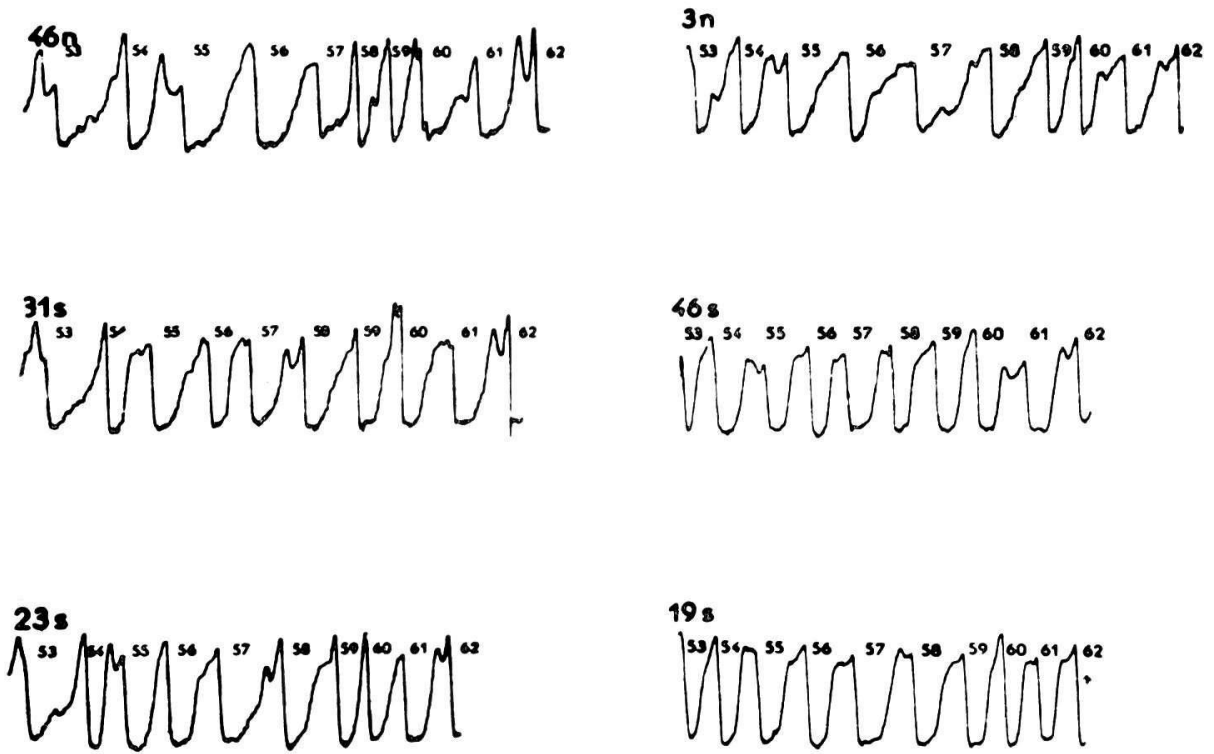

Fig. 5. -- Profils densitométriques de Douglas et de Grandis 
par la largeur des accroissements annuels, qui était précédemment la seule donnée utilisée, aussi bien en dendrochronologie qu'en dendroclimatologie.

Bien entendu, ce sont les variations du climat (s'ajoutant éventuellement à celles de la concurrence entre tiges) qui expliquent ces différences entre critères densitométriques d'une année à l'autre; effectivement, des corrélations très étroites ont pu être mises en évidence entre caractéristiques climatiques et composantes de la densité du bois d'une même année.

Sur la figure 6 sont regroupées les variations de la pluviosité cumulée des mois d'août, septembre et octobre et celles de la densité maximale du Douglas pour les mêmes années; le cœfficient de corrélation était, au cas particulier, de $-0,89$; on peut ainsi prévoir que des pluviosités estivales élevées entraîneront une réduction de densité propre du bois final, et comme conséquence, par exemple, une baisse de résistance mécanique.

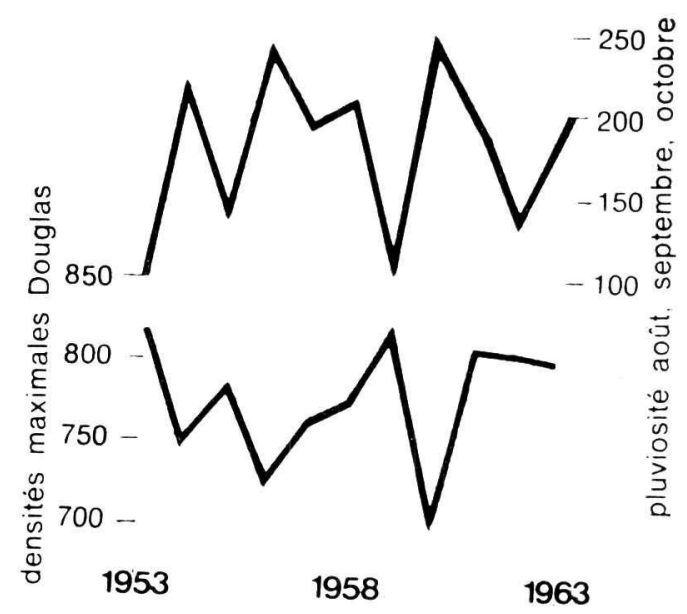

FIG. 6. - Variations des densités maximales en fonction de la pluviosité

\section{2. - Influence des facteurs édaphiques}

\subsection{Expérience sur Pins maritimes en Tunisie.}

Dans le cadre d'une étude plus générale entreprise à la demande de la F.A.O. (Polge et Keller, 1970), un cas d'influence extrêmement marquée du sol sur la qualité du bois a été observé : il s'agissait de deux placeaux situés dans la même parcelle de la forêt de TABARKA, sur le même versant, pente à exposition Nord-Est, à des altitudes assez voisines (165 et $215 \mathrm{~m}$ ) et distants l'un de l'autre de $300 \mathrm{~m}$ environ.

La véritable opposition qui existe au point de vue qualité du bois entre les deux placeaux en cause (placeau 22 et placeau 24) se traduit par le fait que les diagrammes de fréquence, qui ne sont pas très différents pour les largeurs de cernes (les moyennes des deux 
placeaux ne sont pas significativement différentes l'une de l'autre - fig. 7), en arrivent à ne même pas se chevaucher pour les principales caractéristiques technologiques : dureté au torsiomètre (fig. 8), densité (fig. 9) et texture (fig. 10).

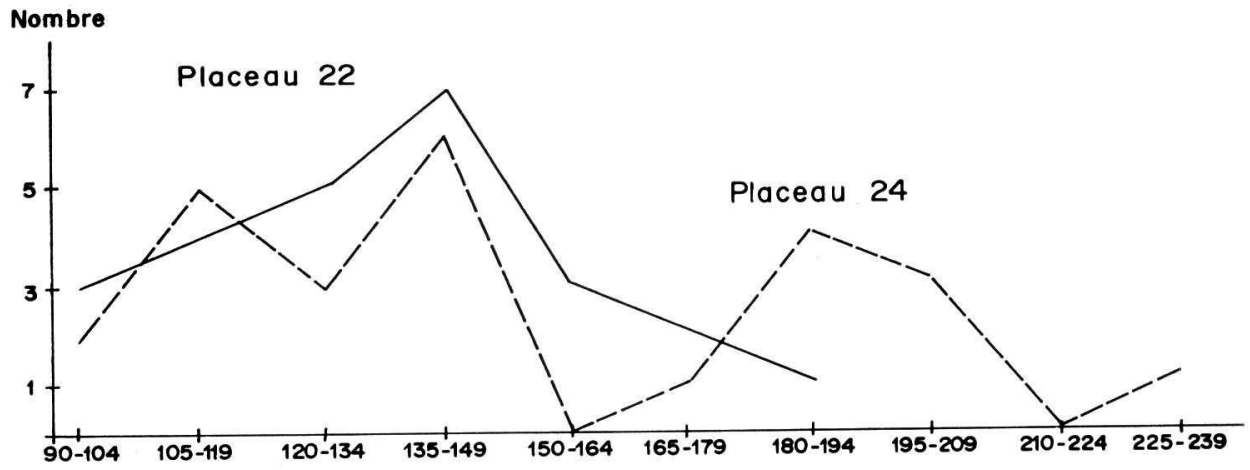

FIG. 7. - Pins maritimes Tabarka - Diagrammes de fréquence des largeurs de cernes

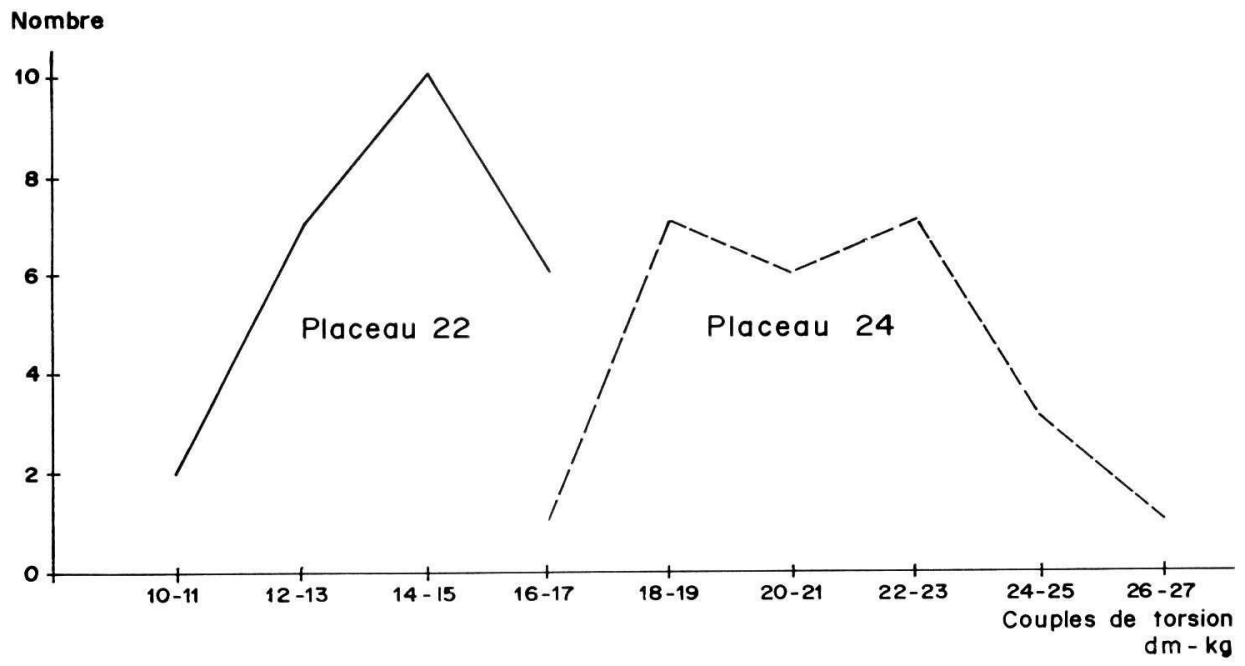

FIG. 8. - Pins maritimes Tabarka - Diagrammes de fréquence des couples de torsion

Le sol des deux stations en cause a été analysé à la diligence de la Station de Recherches sur les Sols Forestiers et la Fertilisation qui a bien voulu également en interpréter pour nous les résultats :

Le sol du placeau 24 a une texture sablo-argileuse ne devenant argileuse qu'à $50 \mathrm{~cm}$ de profondeur; il est, comme celui du placeau 22, légèrement acide ( $\mathrm{pH}$ voisin de 6 ); son complexe absorbant est suffisamment riche en calcium échangeable (entre 1,9 et 3,9 meq/ $100 \mathrm{~g}$ ), mais pauvre en potassium (de $0,1 \mathrm{à} 0,2 \mathrm{meq} / 100 \mathrm{~g}$ ); le rapport $\mathrm{C} / \mathrm{N}$ est très mauvais (37) et peut entraîner des troubles dans l'alimentation azotée des arbres. 


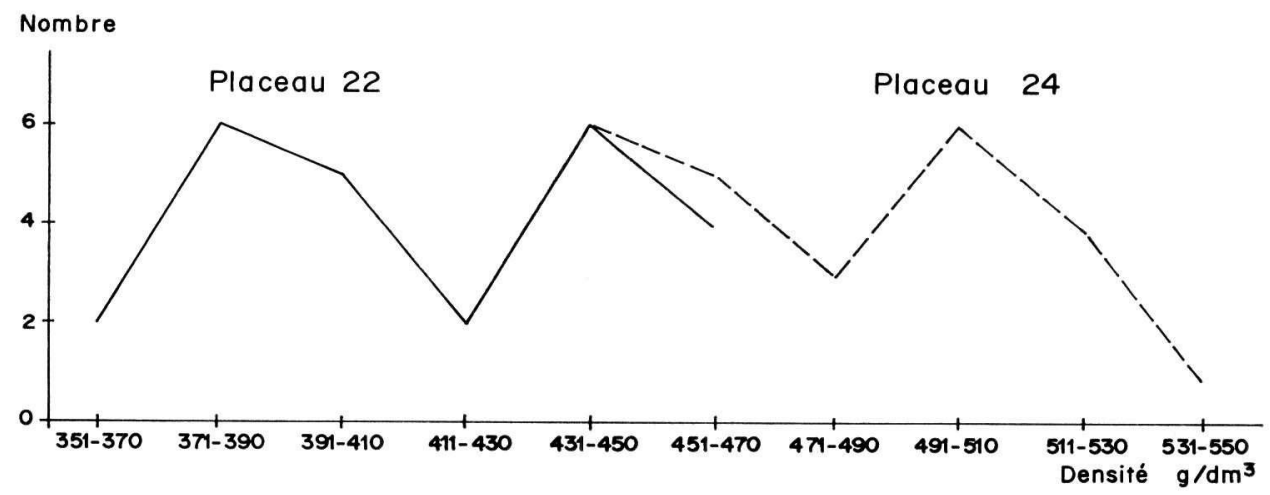

FIG. 9. - Pins maritimes Tabarka - Diagrammes de fréquence des densités

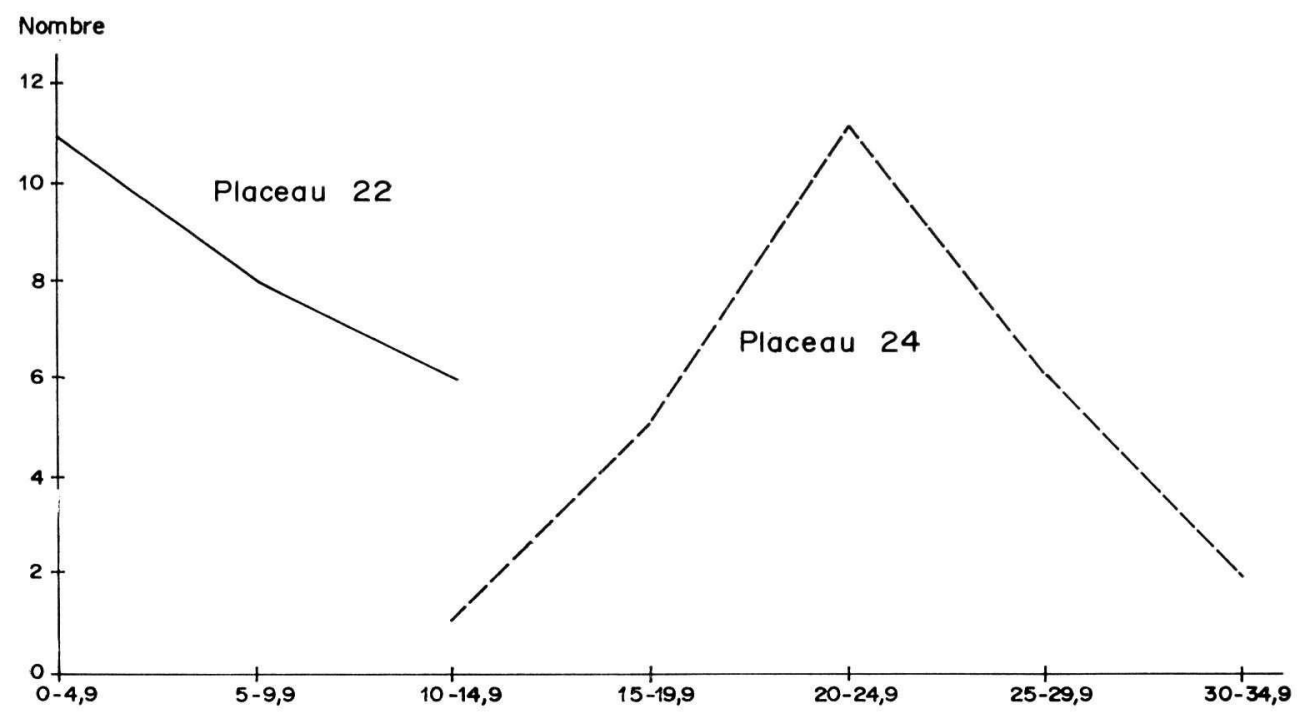

FIG. 10. - Pins maritimes Tabarka - Diagrammes de fréquence des textures

Le sol du placeau 22 est incontestablement plus mauvais que le précédent au point de vue caractéristiques physiques : c'est un sol hydromorphe, extrêmement argileux dans tous ses horizons; en revanche, il est chimiquement plus riche; sa teneur en calcium échangeable est beaucoup plus élevée (de 7,7 meq/100 g), de même que sa teneur en potassium échangeable (de 0,39 à $0,71 \mathrm{meq} / 100 \mathrm{~g}$ ); le rapport $\mathrm{C} / \mathrm{N}$ est normal : 21 en surface, 11 à $50 \mathrm{~cm}$.

Or, à en juger par la largeur des accroissements et la densité du bois, c'est le placeau 24 qui paraît, et de façon très nette, bénéficier des conditions de nutrition les meilleures; on doit en déduire, au cas particulier, que ce n'est pas la richesse du sol qui est en cause, mais sa structure physique; les pluies d'automne notamment, qui représentent $31 \%$ de la pluviosité totale, seraient beaucoup plus bénéfiques sur le sol sableux que sur le sol argileux, d'une part parce que, dans le premier, le tissu radiculaire a plus de facilités pour se développer, 
d'autre part parce que, sur le sol argileux, les premières pluies faisant suite à la sécheresse estivale peuvent se perdre en partie par ruissellement et par réimbibition de l'argile.

Cette expérience a ainsi permis de séparer les effets des caractéristiques physiques et des caractéristiques chimiques du sol; en outre, étant donné que des différences aussi profondes n'ont jamais été observées sous climat tempéré, on peut penser que l'influence des facteurs édaphiques sur la qualité du bois est d'autant plus marquée que les autres conditions de croissance, et notamment celles dues au climat, sont plus défavorables.

\subsection{Effets séparés du sol et de l'hérédité sur les caractéristiques juvéniles du bois de Hêtre.}

Dans le cas précédent, la proximité des deux placeaux en cause et les risques d'hybridation qui en découlent font que les différences constatées peuvent être attribuées de façon à peu près certaine à des différences de sol; mais, lorsqu'on est amené à comparer des peuplements éloignés les uns des autres, il est très difficile d'isoler les effets " facteurs écologiques » des effets génétiques.

C'est ce qui a été tenté dans une expérience afin de faire la part de deux des sources principales de variation de la qualité du bois de Hêtre : sol et hérédité.

Des plants de 3 origines différentes :

- Ligny en Barrois (provenance réputée de bonne qualité),

- Épinal (provenance réputée de médiocre qualité),

- Villers Cotterêts (provenance neutre de réputation moyenne)

ont été installés en bâche dans la pépinière d'Amance sur deux types de sol :

- le sol d'origine de la provenance Ligny en Barrois (désigné ci-dessous sous le nom de sol calcaire),

- le sol d'origine de la provenance Épinal (désigné ci-dessous sous le nom de sol siliceux).

Trois ans après leur mise en place, quelques observations intéressantes ont pu être faites surfun certain nombre de plants enlevés en éclaircie, sans que l'échantillonnage soit suffisant pour pouvoir étudier les interactions sol-hérédité (Polge, Keller et Thiercelin, 1972).

Il apparaît assez clairement que l'effet sol est prépondérant sur les critères de croissance; ainsi, par exemple, pour la circonférence au collet en 1970 (fig. 11), la croissance pour chacune des trois provenances est supérieure sur sol calcaire à ce qu'elle est sur sol silicieux, et la différence est significative pour deux des origines (Ligny et Épinal).

Le problème est plus complexe pour ce qui est de la qualité du bois; si l'on prend pour la caractériser un critère anatomique simple, tel que la porosité (produit du nombre de vaisseaux par leur diamètre moyen), on constate que dans le bois final par exemple (fig. 12), on a certes encore un effet « sol », puisque la porosité de la provenance Ligny en Barrois est plus élevée sur sol calcaire que sur sol silicieux, mais on a, et de façon encore beaucoup plus nette, un effet « hérédité », la porosité décroissant de Villers-Cotterêts à Épinal et à Ligny, que ce soit sur sol calcaire ou sur sol siliceux, où la provenance Ligny se distingue significativement des deux autres. 


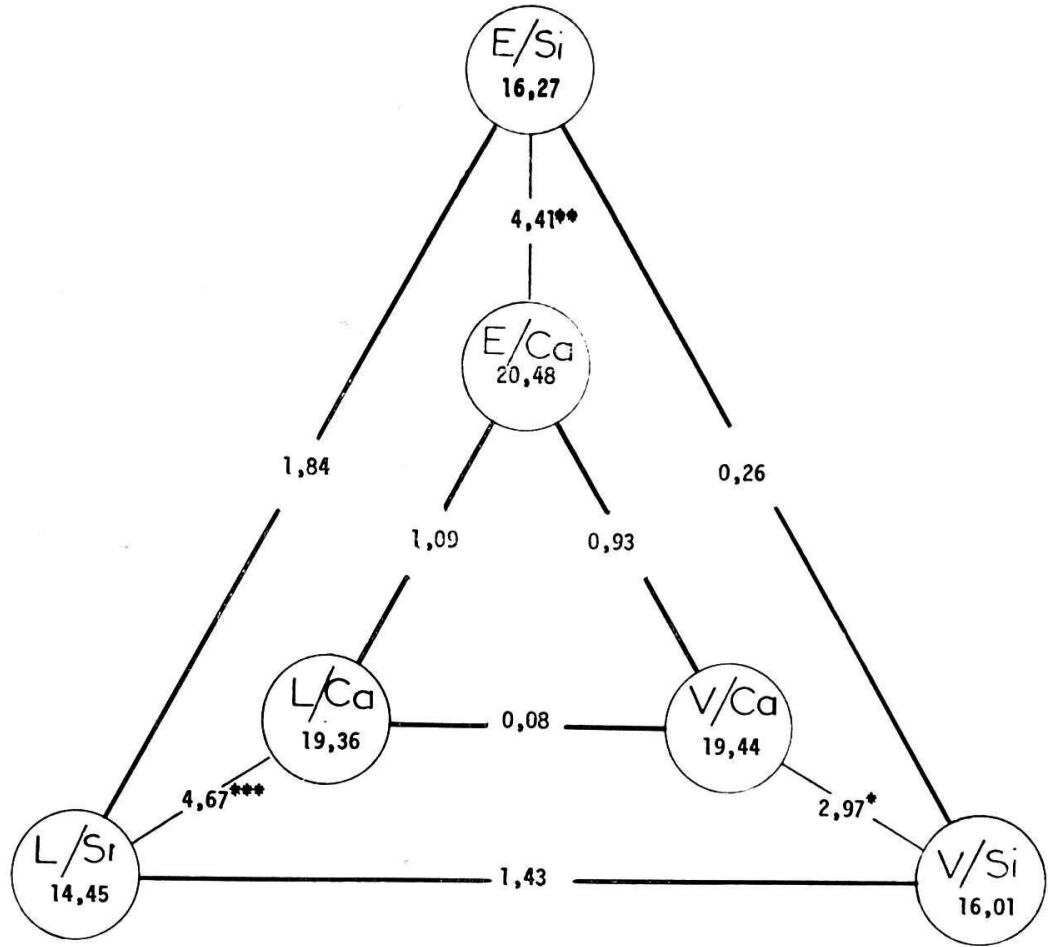

FIG. 11. - Influence de la provenance et du sol sur la croissance. (Circonférence au collet en 1970)

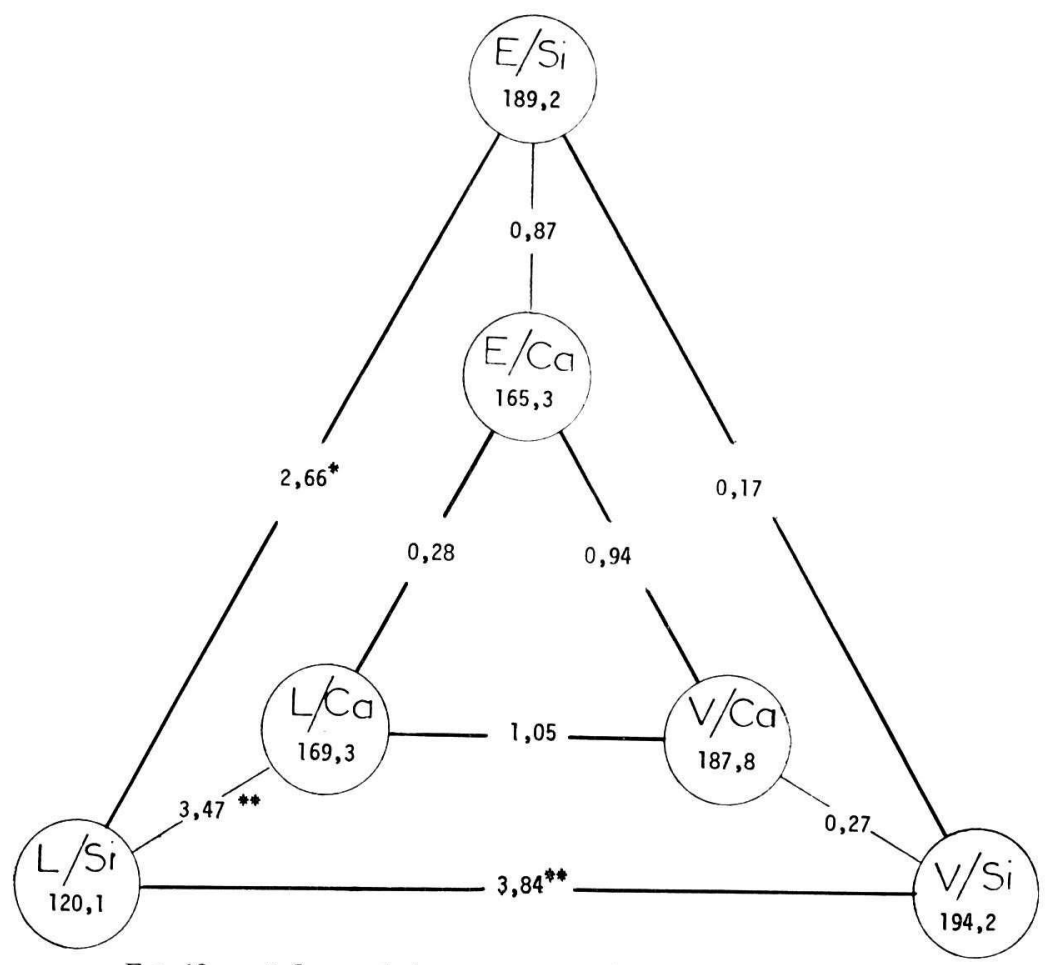

FIG. 12. - Influence de la provenance et du sol sur la qualité du bois. (Nombre de vaisseaux $\times$ Diamètre des vaisseaux) 
2.23. Comparaison de qualité du bois de Sapin pectiné sur roche-mère gréseuse et sur rochemère granitique.

Dans cette expérience en cours d'interprétation des résultats, on a essayé de mettre en évidence l'effet de la roche-mère en sélectionnant deux peuplements aussi voisins que possible au point de vue des conditions de croissance : même altitude, même exposition, même largeur de cernes, même classe de diamètre, même traitement, même densité du peuplement.

Le résultat brut est simple à formuler : contrairement à une opinion répandue parmi les utilisateurs, le sapin poussant sur roche-mère granitique est supérieur pour tout ce qui concerne les critères de densité, donc de résistance mécanique, et la différence avec les sapins poussant sur roche-mère gréseuse est significative pour la densité minimale; cependant, en sus de ce résultat global intéressant par lui-même, cette expérience a permis de mettre en évidence un effet climatique tout à fait inattendu :

Si l'on compare les diagrammes des densités maximales annuelles pour les deux stations en cause de 1930 à 1959 (fig. 13), on constate que les densités maximales sur granite sont supérieures à celles sur grès pour la période comprise entre 1930 et 1946 sans aucune exception; les résultats des deux stations sont à très peu près égaux pour la période 1947 à 1950, et la densité maximale sur grès devient supérieure à celle sur granite pour toute la période 1951 à 1958.

Il y a là un phénomène surprenant dont l'explication a été suggérée par G. AusSENAC sous forme d'une question : « le peuplement voisin de l'une des parcelles en cause n'aurait-il pas disparu par suite d'une opération culturale ou d'un cataclysme naturel, ce qui aurait eu pour conséquence d'entraîner une modification du climat dans le placeau expérimental lui-même? ».

C'est bien ce qui s'est produit puisque le peuplement de sapin sur roche-mère gréseuse était entouré d'épicéas qui ont été détruits progressivement par le bostryche de 1947 à 1950; il s'est produit sur les sapins qui ont subsisté un effet que les bioclimatologistes appellent « effet oasis », se traduisant par une augmentation de l'évapo-transpiration qui a pu entraîner une certaine pénurie d'eau pour les arbres. Or, on a vu qu'une diminution de l'approvisionnement en eau occasionne une hausse des densités maximales annuelles, ce qui se vérifie bien ici puisque cette hausse a été suffisamment importante pour contrebalancer et au-delà l'effet de la roche-mère.

On peut tirer pour conclure de cette expérience deux enseignements de portée générale :

Tout d'abord, elle montre l'intérêt des échanges de vues interdisciplinaires : si l'on n'avait pas eu l'occasion de discuter avec G. AussenAC d'un autre projet commun, on n'aurait sans doute jamais eu l'explication d'un phénomène qui nous déconcertait; c'eut été d'autant plus regrettable que, grâce aux éléments d'information qui nous ont été donnés, une expérience entreprise pour étudier les effets d'un facteur édaphique très précis a permis de déceler en outre une influence très nette de facteurs climatiques.

L'autre leçon est une leçon d'optimisme raisonné : le travail du chercheur est parfois fastidieux, voire décourageant, mais il arrive aussi que la chance lui vienne en aide; c'est ce qui s'est passé dans cette expérience où l'on est parti d'un dispositif expérimental mauvais par suite d'un contrôle insuffisant des sources de variation autres que celles dont on voulait étudier l'effet; mais cette erreur s'est avérée bénéfique, puisqu'il en est résulté des retombées 
Densités maximoles

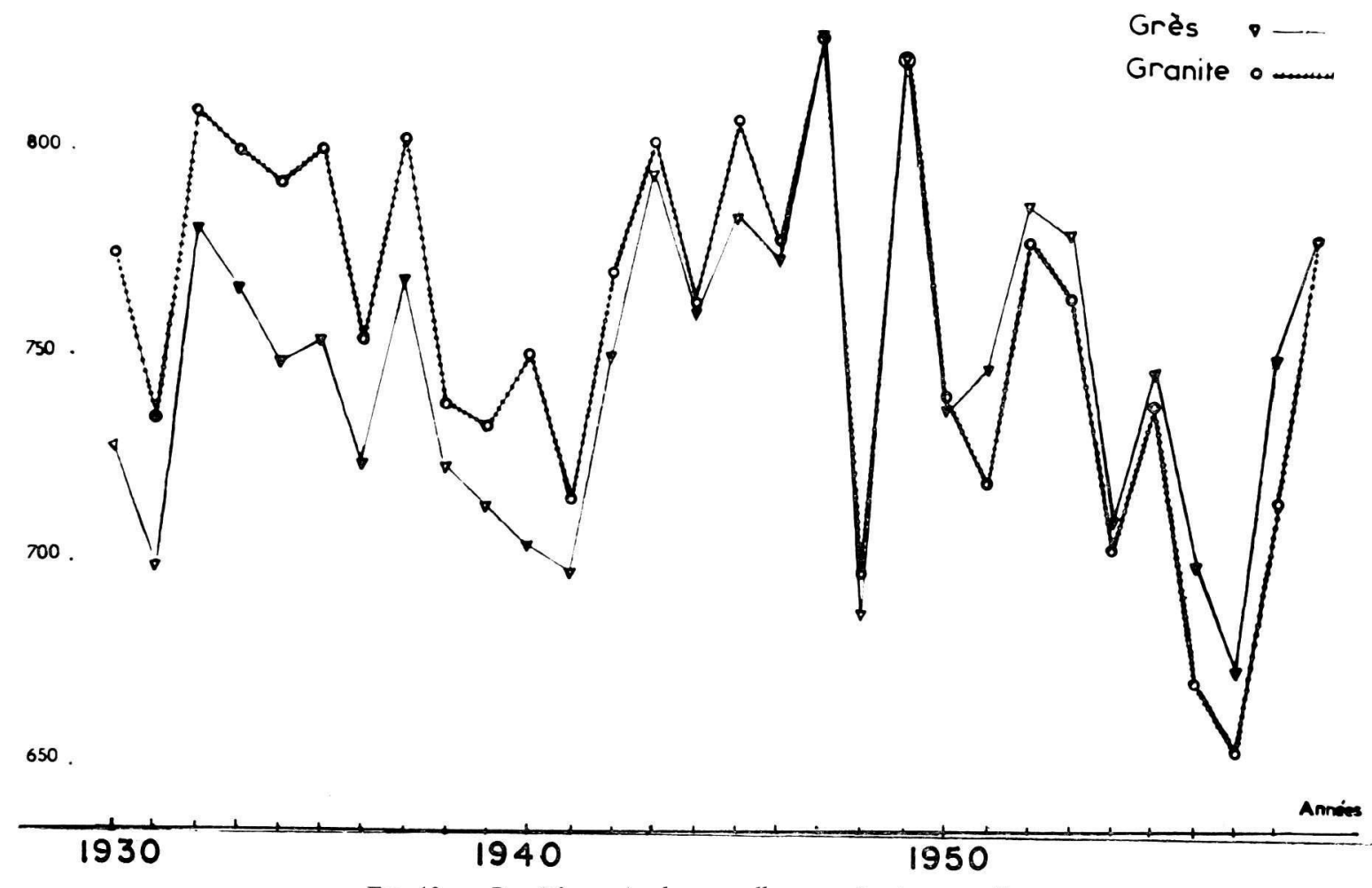

FIG. 13. - Densités maximales annuelles sur grès et sur granite 
qui peuvent intéresser les bioclimatologistes, et puisque, en ce qui nous concerne plus directement, elle a permis de faire ressortir l'extrême sensibilité avec laquelle les composantes de la densité du bois peuvent réagir aux changements qui interviennent dans l'environnement, même relativement lointains, des arbres.

\section{SUMMARY}

\section{ECOLOGICAL FACTORS AND WOOD QUALITY}

\section{1. - Introduction}

The influence of ecological factors on wood quality is so high that the value of timber can change tremendously from one site to the other, e. g. for Quercus petraea and for Quercus robur.

Sometimes the effect of environment is more important than the effect of species, as it is shown by the comparison between Norway Spruce and Scotch Pine of temperate zone and of northern countries (fig. 1).

But generally speaking the influence of species on wood structure is noticeable even when it is low on ringwidth (fig. 2).

Yet it happens that ecological factors have no effect on wood properties of a species or of a group of species (5-needles Pines).

In the other cases, the site conditions change not only the classical wood characteristics but also sometimes other quality criteria (permeability of Douglas fir, red heart of beech).

\section{2. - Some examples of the influence of ecological factors drawn from experiments of the wood quality research station}

\section{1. - Influence of climatic factors :}

- The wood quality of Fagus orientalis increases with elevation due to a decrease of specific gravity and of heterogeneity.

- Because of the wind, the eastern side of Maritime Pine boles in the Landes Forest is made of wood with high density (table 1 and fig. 3), high shrinkage and high lignin content but with short fibers and low pulp yield.

- Irrigation of Scotch Pine during summer increases significantly the width of earlywood without changing anything in the denser part of the ring (table 2),

- The effect of climate on density components is so high that they can fruitfully be used to differenciate the years and to reconstitute the climatic conditions of the past (fig. 4,5 and 6 ).

\section{2. - Influence of edaphic factors :}

From an experiment on Maritime Pine in Tunisia it appears that ringwidth (fig. 7), hardness (fig. 8), density (fig. 9) and latewood percentage (fig. 10) are higher on poor soil with good structure than on rich soil with bad physical characteristics.

Soil also has a clear effect on the ringwidth of young beech seedlings (fig. 11) but both soil and heredity appear to control wood quality (fig. 12).

The density criteria of Silver fir are higher on granite soil than on sandstone, but a so-called " oasis effect » can appear stronger than the soil effect when trees within a close stand become isolated (fig. 13).

\section{RÉFÉRENCES BIBLIOGRAPHIQUES}

Dadswell H. E., 1960. - Tree growth-wood property inter-relationships. Proceedings special field Institute in Forestry Biology.

Hildebrandt G., 1960. - The effect of growth conditions on the structure and properties of wood. Proceedings fifth world forestry congress, vol. 3, août-septembre. 
W. Knigge, 1961. - Kurzfassung der Ergebnisse der Untersuchungen über das Auftreten des Buchenrotkerns in Niedersachsen. Institut für Forstbenutzung der Forstlichen Fakultät der Universität Gottingen.

LARSON P. R., 1960. - A physiological consideration of the springwood summerwood transition in Red pine. Forest Science, vol. 6, $n^{\circ} 2$, June.

LARSON P. R., 1962. - A biological approach to wood quality. Tappi, vol. 45, $n^{\text {o }}$ 6, June.

LARSON P. R., 1963. - The indirect effect of drought on tracheid diameter in red pine. Forest Science, vol. $9, n^{\circ} 1$, March.

Parsa Pajouh D., 1970. - Contribution à l'étude de la qualité du bois de Fagus orientalis de trois stations de la forêt de l'Elbourz au moyen d'essais classiques sur éprouvettes normalisées et d'analyses densitométriques de radiographies. Thèse de Docteur-Ingénieur, Faculté des Sciences de l'Université de Nancy, 16 juillet.

Polge H., Illy G., 1967. - Observations sur l'anisotropie du pin maritime des Landes. Ann. Sci. Forest. 1967, 24 (3), 205-231.

Polge H., Keller R., 1968. - Influence de l'approvisionnement en eau sur la structure interne des accroissements annuels - Expérience d'irrigation sur Pins sylvestres. Ann. Sci. Forest. 25 (3), 125-133.

Polge H., Keller R., 1970. - Rapport de mission de consultants en Tunisie. Document à usage interne Station de Recherches sur la Qualité des Bois $n^{\circ} 4 / 1970$.

Polge H., Keller R., Thiercelin F., 1972. - Effets du sol et de l'hérédité [sur la croissance et les caractéristiques anatomiques de jeunes plants de hêtre. Document à usage interne Station de Recherches sur la Qualité des Bois $n^{\circ} 1 / 1972$.

\section{DISCUSSION ET INTERVENTIONS}

\section{$1^{\circ}$ Questions sur l'anisotropie}

Question de M. Decourt. — « Le fait que les arbres mères de la'lisière Ouest soient plus anisotropes que ceux de la lisière Est ne prouve pas forcément que le vent est responsable, car l'influence du rayonnement solaire n'est pas non plus la même sur les deux lisières »

Réponse : Si l'influence de l'insolation était notable, on aurait une anisotropie NordSud, qui n'existe pas; en fait, Est se distingue significativement des trois autres orientements principaux qui eux-mêmes n'ont pas de différences significatives entre eux.

Question de M. VIART. - «Existe-t-il une influence de l'hérédité sur l'anisotropie? Dans l'affirmative, peut-on l'utiliser pour la sélection de clones de peupliers en vue de l'installation de brise-vents?

Réponse : L'influence de l'hérédité sur l'anisotropie a été étudiée sur le Pin maritime en collaboration avec ILLY; il existe effectivement des différences significatives entre familles, mais rien n'a été fait sur Peuplier.

Question de M. LEMÉE. - « L'anisotropie résultant de fortes pentes a-t-elle fait, pour certaines essences, l'objet de recherches quantitatives? »

Réponse : L'anisotropie due à la pente est importante; elle se traduit par la formation de bois de réaction; par exemple, chez les résineux, les accroissements du côté aval sont plus larges; le bois y est plus dense, mais ses caractéristiques technologiques sont médiocres, en raison en particulier d'une forte rétractibilité axiale; cette anisotropie n'est en général pas attribuée à l'enracinement, mais à un déséquilibre hormonal dû à la dissymétrie du houppier. 


\section{$2^{\circ}$ Question relative à la xylochronologie}

Question de M. BALDY. - «Existe-t-il une liaison entre les caractéristiques de densité et les phénomènes de température? »

Réponse: Il y a une corrélation qui est très nette, en particulier en ce qui concerne les densités maximales annuelles mais, dans tous les cas où elle a été étudiée jusqu'ici, les caractéristiques densitométriques sont moins liées à la température ou à l'insolation qu'à la pluviosité.

\section{$3^{\circ}$ Questions relatives aux liaisons entre largeur des accroissements et qualité du bois}

Question de M. Decourt. - « Les opérations culturales, et notamment les éclaircies entraînent une modification du microclimat; l'effet du traitement en tant que facteur écologique au niveau de l'arbre a-t-il été étudié? »

Réponse: Une seule étude a été faite sans liaison avec les caractéristiques du microclimat dans les placettes à éclaircie forte et à éclaircie faible du Douglas à Saint Just d'Avray; dans cette gamme d'intensités d'éclaircie, il n'y a pas eu d'effet significatif sur la densité $\left(414 \mathrm{~g} / \mathrm{dm}^{3}\right.$ en éclaircie faible contre $411 \mathrm{~g} / \mathrm{dm}^{3}$ en éclaircie forte).

Question de M. Chenal. - «Point actuel des recherches sur la variation des qualités mécaniques en fonction de la largeur des cernes (Exemple des Sapins, Épicéas, Douglas) »

Réponse : Le problème des liaisons entre largeurs de cernes et qualités mécaniques (donc densité) est très complexe :

A l'intérieur d'un même arbre, cette liaison est presque toujours négative, car la densité augmente de la moelle vers l'écorce, alors que la largeur des cernes diminue, et décroît de la base vers le sommet de l'arbre, alors que la largeur des cernes augmente.

Dans des comparaisons faites à âge égal et à même niveau, il convient de distinguer suivant les espèces :

Pour des essences, telles que le Sapin et l'Épicéa par exemple, il y a effectivement en général antinomie entre densité élevée et croissance rapide.

Mais il n'en est pas de même pour le Douglas, où la densité dépend assez peu de la vigueur de croissance, et pour le Pin maritime où les deux données sont liées positivement; pour cette dernière essence, Illy a montré que les familles se différencient surtout entre elles par une croissance plus ou moins prolongée en fin de saison de végétation; ceci explique que les familles à cernes plus larges soient également celles ayant le bois le plus dense, puisque le bois formé en fin de saison de végétation a une densité élevée.

Pour toutes les essences, y compris donc Sapin et Épicéa, il est possible, à l'occasion de comparaisons faites entre provenances, de trouver des provenances ayant à la fois une croissance et une densité satisfaisantes, et il en est de même au niveau individuel.

Question de M. Pourtet. - «Il est très intéressant pour les reboiseurs d'apprendre que des expériences plus poussées montrent que, contrairement aux «on-dit », le Douglas cultivé en France donne du bois de qualité très convenable. Il est probable que la mauvaise impression initiale venait de la comparaison des bois jeunes à croissance rapide de nos reboisements 
avec le bois des peuplements vierges, non éclaircis et pluriséculaires d'Amérique : ceux-ci ont presque disparu maintenant, et les peuplements américains de $2^{\mathrm{e}}$ génération produisent du bois comparable aux nôtres. Le centre Technique du Bois a d'ailleurs démontré que les Douglas du Beaujolais permettaient de fabriquer du contreplaqué de qualités comparables à celui produit par l'Ouest Américain et importé à grands frais ».

Réponse : Oui. Il apparaît effectivement que les Douglas de $2^{\mathrm{e}}$ génération dans l'aire d'origine de cette espèce ne sont supérieurs aux Douglas des reboisements français, ni au point de vue homogénéité, ni au point de vue élagage naturel; l'homogénéité est, après la tardiveté du débourrement le premier critère retenu pour la sélection des Douglas en France.

Question de M. Bouvarel. — «Quelle est l'influence de l'éclaircie sur les composantes de la densité dans le cas du chêne de tranchage?»

Réponse: L'influence de la largeur des accroissements (et non de l'éclaircie) sur les composantes de la densité est actuellement étudiée sur les Chênes de Tronçais : résultats courant 1972.

Question de M. MÉTro. - Étant donné l'importance de la densité comme critère de la qualité du bois, n'est-il pas recommandable de «corréler »systèmatiquement les résultats volumétriques expérimentaux avec la production en matière sèche? ».

Réponse : Oui, c'est certain car, pour toutes les utilisations, ce qui importe, c'est la quantité de membranes cellulaires produites, et non le diamètre des cavités cellulaires, qui conditionne en grande partie le volume apparent; mais, pour le passage de la production en $\mathrm{m}^{3} /$ hectare et par an à la production en tonnes de matière sèche/hectare et par an, il faut connaître, dans chaque cas, les lois de variation de la densité avec la distance à la moelle d'une part, et la hauteur d'autre part. L'équation de la droite de régression, qui permet de passer de la densité mesurée sur une carotte prélevée à hauteur d'homme à la densité globale de l'arbre, présente toujours la même forme, mais les paramètres doivent être ajustés à chaque cas particulier.

Question de M. Venet. - « Les industriels qui déroulent le Pin sylvestre, le Pin maritime ou le Douglas, reprochent à ces bois, moins la largeur des cernes que l'hétérogénéité des bois à cernes larges, non rhédibitoire pour le déroulage mais le rendant plus difficile ».

Réponse : Les études d'héritabilité faites sur Pin maritime ont montré qu'il est vraisemblablement possible de sélectionner, tout au moins pour cette essence, des individus dotés à la fois d'une croissance rapide, d'une densité du bois élevée et d'une bonne homogénéité; un programme spécial d'hybridation est entrepris ce printemps par M. BARADAT dans ce sens.

\section{$4^{\circ}$ Questions relatives à la comparaison grès-granite}

Question de M. Bonneau. - « Les deux peuplements sont-ils à la même altitude? On peut être surpris que, avant isolement, la densité du bois ait pu être plus forte sur granite, alors que les conditions d'alimentation en eau sont vraisemblablement, à priori, meilleures ».

Question de M. Jacamon. - « Même remarque que M. Bonneau. Si l'augmentation de la densité est due, pour le peuplement sur grès, après 1947, à une diminution de l'approvisionnement en eau (évaporation plus forte), je suis étonné que, dans la première phase, 
avant 1947, le peuplement sur granite ait la plus forte densité, l'approvisionnement en eau, sur ce substrat, étant problablement meilleur que sur grès ».

Réponse commune: Les deux peuplements étudiés sont à la même altitude, et toutes les sources de variation autres que la roche-mère ont également été contrôlées en ce qui concerne les placeaux eux-mêmes.

La comparaison ayant été faite sur des arbres de mêmes largeurs de cernes, je ne suis personnellement pas surpris du fait que, avant isolement, la densité du bois sur granite ait été supérieure à celle sur grès, car les conditions de nutrition doivent être prises en considération au même titre que les conditions d'alimentation en eau. 